\title{
(A)symmetry: (Re)inventing the Interdisciplinary Academic Journal
}

A warm welcome to the latest edition of Reinvention: An International Journal of Undergraduate Research.

Six months ago, we promised that Volume 12.2 would be a 'thoroughly interdisciplinary edition, featuring exciting work from a host of international authors' (https://reinventionjournal.org/article/view/441/398). As we have worked to deliver on this promise, the team of staff and students behind Reinvention has been brought to reflect on what it means to produce an interdisciplinary academic journal.

Our editorial team's division of labour means that it is often not until the month of publication that we consider what our newest papers and book reviews might say as a group, and about us. Seeing this edition's titles laid out in front of me, I was struck by the stark, almost symmetrical, divide between papers from the Arts and the Sciences. As you read through this edition, I hope you will pause to reflect on the experience of reading these papers together. There are conversations to be found: James Piggott's and Marin Kuijt's contrasting approaches to reconstructing and reading the past, through archival research and historical video-games, is just one example. This idea of contrast and complementarity was the inspiration for Assistant Editor Kia Yee Ang's beautiful cover design.

In this issue, therefore, we celebrate the collision of arts and science and our goals for a genuinely interdisciplinary academic journal. This concept has been a source of both inspiration and frustration during the production of Reinvention 12.2. It is not uncommon for our international editorial meetings to morph into an existential discussion of what we want the journal to be, and whether our interdisciplinary vision for Reinvention is indeed possible. How can we best accommodate the competing demands of technical precision, reader accessibility and our emphasis on interdisciplinarity? Is any one of these elements more important than another? And, with these goals in mind, how can we best support our authors, many of whom are pursuing a specialist career in academia?

In seeking the answers to these questions, our team has been brought to recognise different forms of symmetry and asymmetry. It seems inevitable that, from time to 
time, the articles we publish will tip the scales in one direction or another: more technical, less readable, more specialised, less interdisciplinary. Yet as we slowly build our repository of undergraduate research, we occasionally gain a glimpse of how this body of work is gradually filling out and finding a kind of symmetry. After 12 years of publication, it's become increasingly common to see submissions from different disciplines that tackle common research problems: social inequality, climate change, human relationships... While it can sometimes be difficult to assure interdisciplinarity within individual articles, our archive as a whole is growing more balanced, more 'symmetrical', with every new paper that arrives in our inbox.

In chasing our goal of interdisciplinarity, we've also learned to look to the world around us. It's important to remember that we are not inventing, but reinventing seeking the marriage of the Arts and Sciences that is abundant in human society. As Rose Hiscock, the Director of Science Gallery Melbourne (an Australian art gallery exploring the boundary between art and science) pointed out in a recent interview, it's a ubiquitous model (https://cosmosmagazine.com/society/when-arts-and-sciencecollide): '[Australian] Aboriginal people have combined science, story-telling, land management, custom, painting, dance and agriculture for more than 50,000 years and today, arts and science remain integral to our lives.' It is the ways in which these seemingly disparate disciplines come together that create a rich and dynamic culture.

Edition 12.2 of Reinvention brings to you five original research papers and one book review that, taken together, reflect the kind of (a)symmetry we are aspiring to.

Temitayo Adeyemi's paper 'A Comprehensive Review on the Potential of Microfabricated Microneedles for Effective Systemic Delivery of Drugs' is an outstanding example of a literature review, an often-overlooked format that we are always keen to publish. By comparing and contrasting the conclusions of different researchers, Adeyemi achieves a novel and robust perspective on the applications of this exciting technology.

In Jack Pearce and Ben Derrick's 'Preliminary Testing: The Devil of Statistics', the authors propose strategies for the improved selection of statistical tests for independent data sets. This paper highlights one of the undergraduate researcher's most valuable assets: the ability to apply 'fresh eyes' to a discipline's entrenched problems that more experienced researchers have learned to take for granted.

The third paper in our 'Sciences' suite is Abraham Herzog-Arbeitman's 'Polyelectrolyte Complexation: Entropy, Medicine and the Beginning of Life'. Drawing together the 
application of polyelectrolyte complexes in medicine and their possible role in life formation, Herzog-Arbeitman's research is exactly the kind of interdisciplinary article our journal aspires to.

These papers are mirrored by our two excellent 'Arts' articles and book review.

James Piggott's 'The Impact of Censorship on the "Historical" Video-Game' tackles the implications of the growing use of video-games as tools of historical education and research. It seems fitting that such an impressive paper discussing the convergence of the arts and sciences should find a place in this edition.

This brings me to the final paper of this edition, Marin Kuijt's 'Exposing the Colonial Exhibition: Dutch Anti-Colonial Activism in a Transnational Context'. It's always a thrill to hear from established peer reviewers how an undergraduate research paper represents a genuine intervention in their field. This article received glowing reviews, and we are proud to include it in Reinvention.

Finally, Dr Kristen Davis and Anna Bray's two-part review of Vanessa Panfil's 2017 book The Gang's All Queer: The Lives of Gay Gang Members is an incisive reflection on a ground-breaking text. Davis is a lecturer in criminology at Monash University; Bray is a student of sociology at the University of Warwick. Their combined review is a testament to the merits of an interdisciplinary approach.

To those who may be reading this and contemplating submitting to Reinvention, a few words of advice. In collaboration with our future authors, we want to take the next step towards our goal of a genuinely interdisciplinary academic journal. As well as bringing together articles from disparate disciplines, we are seeking to publish articles that feature interdisciplinary research. Even during the earliest stages of research, you should be thinking about the implications of your work beyond your own discipline. A close awareness of these larger dimensions gives you the strongest possible chance of achieving publication in our journal.

A huge thanks to everyone who worked to produce this issue of Reinvention. Our international editorial board - Alex, Connie, Kia Yee, Millie and Polina in Warwick along with Raphael, Madeleine, Sarsha and Tiana here in Melbourne - your clearsightedness and commitment to our authors is always inspiring. Fiona, Emma, Victoria and Kate - thank you for your support, and your confidence. 
Although I am signing off here, I have great pleasure in handing over to incoming Editor, Polina Zelmanova. Polina has shown great leadership as a member of the editorial team, and I'm excited to watch her lead the journal in a new direction.

Thank you - and happy reading!

To cite this paper please use the following details: Duffus, H. (2019), '(A)symmetry: (Re)inventing the Interdisciplinary Academic Journal', Reinvention: an International Journal of Undergraduate Research, Volume 12, Issue 2, https://reinventionjournal.org/article/view/543. Date accessed [insert date]. If you cite this article or use it in any teaching or other related activities please let us know by emailing us at Reinventionjournal@warwick.ac.uk. 